Z. Klin. Chem. Klin. Biochem.

13. Jg. 1975, S. 417-421

\title{
Quantitative Bestimmung der enteralen Calciumresorption mit angereichertem stabilem ${ }^{46} \mathrm{Ca}$
}

\author{
Von M. Persigehl, K. Kasperek, F. Ritzl, A. Höck und L. E. Feinendegen
}

Institut für Medizin, Kernforschungsanlage Jülich $\mathrm{GmbH}$,Jülich

(Eingegangen am 23. April 1974/2. Juni 1975)

Zusammenfassung: Die Anwendbarkeit eines Ca-Resorptionstestes ohne Gebrauch von Radioisotopen, der somit auch bei Säuglingen, Kindern und Schwangeren möglich ist, wurde bei 14 erwachsenen Patienten untersucht. Anstelle eines Radioisotops wurde das stabile ${ }^{46} \mathrm{Ca}$ in angereicherter Form oral verabreicht und die prozentualen Anteile der applizierten Dosis an ${ }^{46} \mathrm{Ca}$ im Serum und im Urin gemessen. Die Messung des ${ }^{46} \mathrm{Ca}$ erfolgte nach Aktivierung mit thermischen Neutronen über das ${ }^{47} \mathrm{Sc}$. Es konnte gefunden werden, daß das klinische Bild weitgehend mit den ermittelten Resorptionsgrößen übereinstimmt. Anhand der Ergebnisse lassen sich drei Patientengruppen unterscheiden, und zwar Patienten mit normaler, vermehrter und verminderter Ca-Resorption.

\section{Quantitative determination of enteral calcium resorption with enriched stable ${ }^{46} \mathrm{Ca}$}

Summary: The application of a Ca-resorption test without the use of radioisotopes, which can therefore be used for babies, children and pregnant women, was investigated on 14 adult patients. In place of a radioisotope, an enriched form of the stable isotope ${ }^{46} \mathrm{Ca}$ was administered orally, and the proportion of the applied dose of ${ }^{46} \mathrm{Ca}$ appearing in the serum and urine was measured. ${ }^{46} \mathrm{Ca}$ was measured by neutron activation over ${ }^{47} \mathrm{Sc}$. The clinical picture showed a close correlation with the determined resorption capacities. On the basis of the results, patients were classified into three groups: those with normal, increased, and decreased Ca-resorption.

\section{Einleitung}

Die enterale Ca-Resorption, die bei einer Reihe von Erkrankungen von großem Interesse ist, wurde bislan 3 vornehmlich mittels oraler Zufuhr radioaktiver $\mathrm{Ca}$ Isotope überprüft (1-4). So wurde z. B. von Avioli (5) nach oraler Zufuhr von ${ }^{47} \mathrm{Ca}$ die enterale Resorption dieses Isotops über einen Zeitraum von 4 Stunden durch Bestimmung der Aktivität im Serum gemessen. Derartige Resorptionsbestimmungen sind bei einer Gruppe von Patienten - Kindern, Jugendlichen, Schwangeren - wegen der damit verbundenen Strahlenbelastung nur unter sehr großen Vorbehalten durchführbar. Die Nutzung stabiler Isotope ermöglicht gerade bei diesem Personenkreis die Prüfung des hier häufig gestörten $\mathrm{Ca}$ Stoffwechsels (6-11).

Neben den Radioisotopen haben fast alle Elemente mehrere stabile Isotope, die für jedes Element in einem charakferistischen Mischungsverhältnis vorliegen. So setzt sich das natürliche $\mathrm{Ca}$ aus den folgenden 6 stabilen Isotopen zusammen:

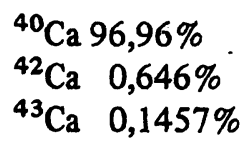

$$
\begin{aligned}
& { }^{44} \mathrm{Ca} 2,06 \% \\
& { }^{46} \mathrm{Ca} 0,0033 \% \\
& { }^{48} \mathrm{Ca} 0,185 \%
\end{aligned}
$$

Mc Pherson (6) konnte zeigen, daß die einzelnen CaIsotope vom menschlichen Organismus gleichartig behandelt werden. Ändert man nun die von $A$ violi benutzte Versuchsanordnung so um, daß anstelle des Radioisotops ein an- oder abgereichertes stabiles Isotop verabreicht wird und mißt die Menge des oral zugeführten stabilen Isotops nach Verteilung im Serum mit Hilfe der Neutronenaktivierungsanalyse, so ist dieser Resorptionstest auch bei der oben genannten Patientengruppe möglich (7). Die Brauchbarkeit dieses Untersuchungsverfahrens wurde in der vorliegenden Arbeit bei 14 Patienten weiter geprüft.

\section{Material und Methoden}

Bei den 14 untersuchten Patienten, 2 Männern und 12 Frauen im Alter von 21 bis $65 \mathrm{Jahren,} \mathrm{lag} \mathrm{in} 9$ Fällen kein Anhalt für eine Störung des $\mathrm{Ca}-\mathrm{Stoff}$ wechsels vor. Bei den übrigen 5 Patienten waren entweder aus klinischer Sicht oder mit Hilfe von Laboruntersuchungen Hinweise für eine Störung des CaStoffwechsels vorhanden (siehe Tab. 1). Bei allen Patienten wurde je ein Resorptionstest nach 3-tägiger Gabe einer Caarmen Kost und dann nach 4-tägiger Zufuhr einer $\mathrm{Ca}$-reichen Nahrung durchgefüht. In der Ca-armen Diät wurden alle $\mathrm{Ca}$ reichen Nahrungsbestandteile wie z. B. Milch oder Milchprodukte weggelassen, so daß die tägliche $\mathrm{Ca}$-Zufuhr durchschnittlich $440 \mathrm{mg}$ betrug. Die Ca-reiche Diät setzte sich zusammen 
Tab. 1. Angaben zu den 14 untersuchten Patienten

\begin{tabular}{|c|c|c|c|c|c|c|}
\hline Lfd. Nr. & Name & Altcr & $\begin{array}{c}\text { Geschlecht } \\
\text {. }\end{array}$ & $\begin{array}{l}\text { Serum-Ca } \\
(\mathrm{mmol} / \mathrm{l})\end{array}$ & Ca-Resorption & Diagnosen \\
\hline 1 & M. Z. & 21 & q & 2,33 & normal & Zustand nach Thyreoidektomie \\
\hline 2 & K. K. & 29 & $\varphi$ & 2,35 & normal & Zungengrundstruma \\
\hline 3 & G. B. & 55 & \& & 2,38 & normal & Diabetes mellitus \\
\hline 4 & F. G. & 23 & $\delta$ & 2,13 & normal & Hyperventilationstetanie \\
\hline 5 & T. L. & 32 & \& & 2,35 & normal & Struma parenchymatosa \\
\hline 6 & J. N. & 41 & \% & 2,35 & normal & Vegetative Dystonie \\
\hline 7 & T. R. & 36 & ९ & 2,23 & normal & Hyperventilationstetanie \\
\hline 8 & H. K. & 34 & 2 & 2,50 & normal & Struma nodosa \\
\hline 9 & G. B. & 65 & o & 2,35 & erhöht & sek. Hyperparathyreoidismus \\
\hline 10 & R. K. & 27 & 8 & 2,00 & erhöht & Tetanie \\
\hline 11 & H. M. & 34 & @ & 2,35 & erhöht & Tetanie \\
\hline 12 & M. M. & 35 & 9 & 2,30 & erhöht & Tetanie \\
\hline 13 & A. B. & 55 & \& & $2,00^{\circ}$ & erniedrigt & anazide Gastitis \\
\hline 14 & H. T. & 30 & 8 & 2,15 & erniedrigt & endogene Depression \\
\hline
\end{tabular}

aus der gleichen Ca-armen Grundnahrung, zuzüglich 2 CaBrausetabletten pro Tag, die zusammen $5880 \mathrm{mg}$ Calciumlactatgluconat und $600 \mathrm{mg}$ Calciumcarbonat enthielten, entsprechend etwa $1000 \mathrm{mg}$ ionisiertem Ca.

Vor der Durchführung des Rcsorptionstestes wurde ein mit ${ }^{46} \mathrm{Ca}$ angereichertes $\mathrm{Ca}$-Isotopengemisch, in dem das $\mathrm{Ca}$ als $\mathrm{CaCO}_{3}$ vorlag, durch $\mathrm{Zufuhr}$ von $\mathrm{HCl}$ in $\mathrm{CaCl}_{2}$ überführt. Dieses $\mathrm{CaCl}_{2}$ wurde in $20 \mathrm{ml}$ Wasser gelöst und den nüchternen Patienten morgens als Getränk gegeben. Die durchschnittliche Menge an ${ }^{46} \mathrm{Ca}$ betrug $0,1476 \mathrm{mg}$, die Gesamt-Ca-Dosis schwankte zwischen 3,3 und 0,36 mg; der relative Anteil des angereicherten ${ }^{46} \mathrm{Ca}$ reichte von 4,8 bis $30,94 \%$, dies entspricht einem Anreicherungsfaktor gegenüber dem normalen Isotopengemisch von 1454 bis 9375 .

Von allen Patienten wurden venöse Blutproben 0,5, 1, 2, 4, 8, 16, 24 Stunden nach $\mathrm{Ca}-\mathrm{Gabe}$ entnommen. Gleichzeitig wurde über 24 Stunden der gesamte Urin gesammelt. Nach dem Zentrifugieren des Blutes wurde das $\mathrm{Ca}$ in $3 \mathrm{ml}$ Serum als $\mathrm{Ca}(\mathrm{OOC})_{2}$ gefällt (12). Der so gewonnene Niederschlag wurde nach Trocknung in ein Polyäthylenröhrchen eingeschweißt und im Reaktor gleichzeitig mit einer entsprechenden Vergleichsprobe $\mathrm{CaCO}_{3}$ (Standard) mit thermischen Neutronen aktiviert. Das $\mathrm{Ca}$ im Urin wurde aus einer 10-ml-Probe mit Oxalsäure gefällt (12) und nach Trocknung und Einschweißung ebenfalls im Reaktor aktiviert. Dabei bilden sich einmal aus dem stabilen angereicherten ${ }^{46} \mathrm{Ca}$ - das Radioisotop ${ }^{47} \mathrm{Ca}$ sowie aus dem im normalen $\mathrm{Ca}-\mathrm{Gemisch}$ enthaltenen ${ }^{48} \mathrm{Ca}$ das ${ }^{49} \mathrm{Ca}$. Die hier interessierenden radioaktiven Ca-Isotope zerfallen wie folgt:

$\begin{array}{lll}{ }^{47} \mathrm{Ca} \frac{\beta, \gamma}{4,7 \mathrm{~d}} & { }^{47} \mathrm{Sc} \frac{\beta, \gamma}{3,4 \mathrm{~d}} & { }^{47} \mathrm{Ti} \\ { }^{49} \mathrm{Ca} \frac{\beta, \gamma}{8,8 \mathrm{~m}} & { }^{49} \mathrm{Sc} \frac{\beta}{58 \mathrm{~m}} & { }^{49} \mathrm{Ti}\end{array}$

5-6 Tage nach Bestrahlungsende erreicht die Radioaktivität des aus dem ${ }^{47} \mathrm{Ca}$ entstehenden ${ }^{47} \mathrm{Sc}$ ein Maximum und ist dann über die 0,16-MeV-Linie sehr gut meßbar. Die Radioaktivität des in diesem Energiebereich zunächst stark störenden ${ }^{24} \mathrm{Na}$ mit einer Halbwertszeit von 15 Stunden ist bis dahin weitgehend abgeklungen. Das ${ }^{49} \mathrm{Ca}$ wird unmittelbar nach Bestrahlungsende am Reaktor über die 3,1-MeV-Linie gemessen.

Der prozentuale Anteil x des applizierten Marker-Elementes pro Liter Serum wird wie folgt berechnet (7):

$x=\left(\frac{W}{G}\right) \cdot \frac{\left(\frac{Q_{2}}{P_{2}}\right)-\left(\frac{Q_{1}}{P_{1}}\right)}{\left(\frac{K_{2}}{h_{2}}\right)-\left(\frac{K_{1}}{h_{1}}\right)} \cdot\left(\frac{V}{V_{p}}\right) \cdot 100[\%]$ wobei

$\mathrm{W}=$ Gewicht der Vergleichsprobe $[\mathrm{g}]$

$\mathrm{G}_{0}=$ Gewicht der Gesamtmenge des applizierten Elementes [g]

$\mathrm{Q}_{1}=$ Intensität der Gammastrahlung mit der Energie $\mathrm{E}_{1}(0,16$ $\mathrm{MeV}$ ) der Probe

$\mathrm{Q}_{2}=$ Intensität der Gammastrahlung mit der Energie $\mathrm{E}_{2}(3,1$ $\mathrm{MeV}$ ) der Probe

$P_{1}=$ Intensität der Gammastrahlung mit der Energie $E_{1}(0,16$ $\mathrm{MeV}$ ) der Vergleichsprobe

$P_{2}=$ Intensität der Gammastrahlung mit der Energie $E_{2}$ (3,1 $\mathrm{MeV}$ ) der Vergleichsprobe

$K_{1}=$ Anteil des Isotops $B_{1}$ im Element mit dem angereicherten Isotop $\mathrm{B}_{2}$

$\mathrm{K}_{\mathbf{2}}=$ Anteil des Isotops $\mathrm{B}_{\mathbf{2}} \mathrm{im}$ Element mit dem angereicherten Isotop $\mathrm{B}_{2}$

$\mathrm{h}_{1}=$ Anteil des Isotops $B_{1}$ im Element mit natürlicher Isotopenzusammensetzung

$\mathrm{h}_{2}$ Anteil des Isotops $\mathrm{B}_{\mathbf{2}} \mathrm{im}$ Element mit natürlicher Isotopenzusammensetzung

$V=$ Bezugsvolumen - z. B. 11 - (bzw. Bezugsgewicht)

$\mathrm{V}_{\mathrm{p}}=$ Volumen (bzw. Gewicht) der aktivierten Proben [ml]

Bei 7 Patienten wurde neben dem angereicherten ${ }^{46} \mathrm{Ca}$ gleichzeitig ${ }^{45} \mathrm{Ca}$ oral verabreicht und bei gleicher Probenentnahme mittels Messung im Flüssigkeitsszintillationszähler auch der Anteil des resorbierten ${ }^{45} \mathrm{Ca}$ bestimmt. Die so für beide Ca-Isotope ermittelten Werte stimmten innerhalb einer Fehlerbreite von $\pm 5,8 \%$ beim ${ }^{45} \mathrm{Ca}$ und $\pm 3,6 \%$ beim stabil markierten ${ }^{46} \mathrm{Ca}$ überein.

\section{Ergebnisse}

Die zu den verschiedenen Zeiten nach oraler Zufuhr von Ca mit stabil markiertem ${ }^{46} \mathrm{Ca}$ ermittelten prozentualen Anteile der applizierten Menge an ${ }^{46} \mathrm{Ca}$ pro 1 Serum sind in Tabelle 2 und 3 zusammengestellt. Dabei gibt Tabelle 2 die Werte nach 3-tägiger Ca-armen Diät und Tabelle 3 die Daten nach 4-tägiger Ca-reicher Kost wieder. $\mathrm{Da}$ die $\mathrm{Ca}$-Resorption im wesentlichen in den ersten 2 Stunden nach Aufnahme stattfindet und laut Birge et al. (2) dann zu über $95 \%$ abgeschlossen ist, wurden von den Ergebnissen bei $1 / 2 \mathrm{~h}, 1 \mathrm{~h}$ und $2 \mathrm{~h}$ die Mittelwerte gebildet und Resorptionsmittelwerte genannt. Diese wurden arbitrarisch in drei Gruppen unterteilt. Im Versuch bei Ca-armer Diät (Tab. 2) um- 
Tab. 2. Prozentuale Anteile und „Resorptionsmittelwerte“ der applizierten Dosis ${ }^{46}$ Ca pro 1 Serum und im $24 \mathrm{~h}$-Urin zu verschiedenen Zeiten nach oraler Zufuhr des Isotopengemischs nach Ca-armer Kost, unterteilt in Gruppen mit vermehrter (I), normaler (II) und verminderter (III) Resorption.

\begin{tabular}{|c|c|c|c|c|c|c|c|c|c|c|}
\hline \multirow[t]{3}{*}{ Gruppe } & \multirow[t]{3}{*}{ Patient } & \multicolumn{7}{|c|}{$\begin{array}{l}\% \text { der applizierten Dosis }{ }^{46} \mathrm{Ca} / \mathrm{l} \text { Serum zu verschiedenen Zeiten nach } \\
\text { Ca-Zufuhr }(\mathrm{h})\end{array}$} & \multirow{3}{*}{$\begin{array}{l}\text { Resorptions- } \\
\text { mittelwerte } \\
\frac{x_{1}+x_{2}+x_{3}}{3}\end{array}$} & \multirow{3}{*}{$\begin{array}{l}\% \text { der appli- } \\
\text { zierten Dosis } \\
{ }^{46} \mathrm{Ca} \text { im } \\
24 \mathrm{~h} \text {-Urin }\end{array}$} \\
\hline & & 0,5 & 1 & 2 & 4 & 8 & 16 & 24 & & \\
\hline & & $x_{1}$ & $x_{2}$ & $x_{3}$ & $x_{4}$ & $x_{5}$ & $x_{6}$ & $x_{7}$ & & \\
\hline 1 & $\begin{array}{l}\text { G. B. } \\
\text { R. K. } \\
\text { H. M. } \\
\text { M. M. }\end{array}$ & $\begin{array}{l}0,6 \\
3,0 \\
1,3 \\
1,8\end{array}$ & $\begin{array}{l}5,6 \\
2,5 \\
2,3 \\
2,0\end{array}$ & $\begin{array}{l}3,4 \\
1,8 \\
2,9 \\
2,2\end{array}$ & $\begin{array}{l}2,8 \\
1,0 \\
1,7 \\
1,2\end{array}$ & $\begin{array}{l}0,9 \\
1,4 \\
1,4 \\
1,1\end{array}$ & $\begin{array}{l}0,6 \\
1,2 \\
1,9 \\
1,2\end{array}$ & $\begin{array}{l}0,5 \\
1,0 \\
1,8 \\
0,2\end{array}$ & $\begin{array}{l}3,2 \\
2,76 \\
2,16 \\
2,0\end{array}$ & $\begin{array}{l}1,6 \\
1,7 \\
2,7 \\
5,2\end{array}$ \\
\hline II & $\begin{array}{l}\text { M. Z. } \\
\text { K. K. } \\
\text { G. B. } \\
\text { F. G. } \\
\text { T. L. } \\
\text { J. N. } \\
\text { T. R. } \\
\text { H. K. }\end{array}$ & $\begin{array}{l}1,0 \\
1,6 \\
1,4 \\
1,3 \\
1,6 \\
1,3 \\
1,1 \\
0,2\end{array}$ & $\begin{array}{l}1,8 \\
1,8 \\
1,1 \\
1,5 \\
1,6 \\
1,5 \\
1,3 \\
0,6\end{array}$ & $\begin{array}{l}1,7 \\
2,0 \\
0,9 \\
1,7 \\
1,5 \\
1,6 \\
1,2 \\
1,4\end{array}$ & $\begin{array}{l}1,5 \\
1,6 \\
1,2 \\
1,5 \\
1,4 \\
1,3 \\
1,3 \\
1,5\end{array}$ & $\begin{array}{l}1,7 \\
1,3 \\
1,4 \\
1,3 \\
1,0 \\
1,3 \\
1,0 \\
1,0\end{array}$ & $\begin{array}{l}1,1 \\
1,3 \\
0,4 \\
1,0 \\
0,9 \\
1,0 \\
0,8 \\
1,1\end{array}$ & $\begin{array}{l}0,9 \\
0,1 \\
0,6 \\
0,9 \\
0,7 \\
0,8 \\
0,6 \\
1,0\end{array}$ & $\begin{array}{l}1,5 \\
1,8 \\
1,13 \\
1,5 \\
1,56 \\
1,46 \\
1,2 \\
0,73\end{array}$ & $\begin{array}{l}2,2 \\
5,7 \\
0,5 \\
1,2 \\
2,4 \\
1,7 \\
1,2 \\
2,5\end{array}$ \\
\hline 111 & $\begin{array}{l}\text { A. B. } \\
\text { H. T. }\end{array}$ & $\begin{array}{l}0,6 \\
0\end{array}$ & $\begin{array}{l}0,2 \\
0\end{array}$ & $\begin{array}{l}0,3 \\
0,2\end{array}$ & $\begin{array}{l}0,1 \\
0,3\end{array}$ & $\begin{array}{l}0,1 \\
0,1\end{array}$ & $\begin{array}{l}0,1 \\
0\end{array}$ & $\begin{array}{l}0 \\
0\end{array}$ & $\begin{array}{l}0,36 \\
0,1\end{array}$ & $\begin{array}{l}0,2 \\
1,1\end{array}$ \\
\hline
\end{tabular}

Tab. 3. Prozentuale Anteile und „Resorptionsmittelwerte“ der applizierten Dosis ${ }^{46} \mathrm{Ca}$ pro I Serum und im 24 h-Urin zu verschiedenen Zeiten nach oraler Zufuhr des Isotopengemischs nach Ca-reicher Kost, unterteilt in Gruppen mit vermchrter (I), normaler (II) und verminderter (III) Resorption.

\begin{tabular}{|c|c|c|c|c|c|c|c|c|c|c|}
\hline \multirow[t]{3}{*}{ Gruppe } & \multirow[t]{3}{*}{ Patient } & \multicolumn{7}{|c|}{$\begin{array}{l}\text { \% der applizierten Dosis }{ }^{46} \mathrm{Ca} / 1 \text { Serum zu verschiedenen Zeiten nach } \\
\text { Ca-Zufuhr }(\mathrm{h})\end{array}$} & \multirow{3}{*}{$\begin{array}{l}\text { Resorptions- } \\
\text { mittelwerte } \\
\frac{x_{1}+x_{2}+x_{3}}{3}\end{array}$} & \multirow{3}{*}{$\begin{array}{l}\text { \% der appli- } \\
\text { zierten Dosis } \\
{ }^{46} \mathrm{Ca} \text { im } \\
24 \mathrm{~h} \text {-Urin }\end{array}$} \\
\hline & & 0,5 & 1 & 2 & 4 & 8 & 16 & 24 & & \\
\hline & & $x_{1}$ & $x_{2}$ & $x_{3}$ & $x_{4}$ & $x_{5}$ & $x_{6}$ & $x_{7}$ & & \\
\hline \multirow[t]{4}{*}{ I } & G. B. & 2,7 & 4,0 & 4,2 & 4,0 & 3,1 & 0,7 & 0 & 3,6 & 2,4 \\
\hline & R. K. & 2,4 & 4,1 & 3,0 & 3,0 & 1,8 & 1,2 & 1,0 & 3,2 & 3,8 \\
\hline & H. M. & 3,9 & 3,7 & 2,3 & 2,0 & - & 2,2 & 2,6 & 3,3 & 4,8 \\
\hline & M. M. & 4,5 & 3,7 & 2,6 & 1,7 & 2,1 & 0,8 & 1,6 & 3,6 & 6,3 \\
\hline \multirow[t]{8}{*}{ II } & M. Z. & 1,6 & 2,3 & 2,6 & 2,3 & 1,2 & 1,1 & 1,2 & 2,16 & 3,9 \\
\hline & K. K. & 2,0 & 3,0 & 3,0 & 3,0 & 1,9 & 0,8 & 0,2 & 2,66 & 4,2 \\
\hline & G. B. & 0,9 & 0,7 & 2,3 & 1,6 & 1,8 & 0,5 & 0,7 & 1,3 & 1,6 \\
\hline & F. G. & 1,7 & 2,2 & 2,3 & 1,8 & 1,7 & 1,3 & 0,8 & 2,06 & 3,7 \\
\hline & T. L. & 1,2 & 1,9 & 2,0 & 1,7 & 1,6 & 1,1 & 0,8 & 1,7 & 2,6 \\
\hline & J. N. & 2,0 & 2,2 & 2,1 & 1,6 & 1,4 & 1,3 & 0,9 & 2,1 & 3,1 \\
\hline & T. $\mathbf{R}$. & 1,5 & 2,2 & 2,0 & 1,8 & 1,5 & 1,1 & 0,8 & 1,9 & 3,2 \\
\hline & H. K. & 1,8 & 2,2 & 2,7 & 3,0 & 2,6 & 0,6 & 0,3 & 2,33 & 5,7 \\
\hline \multirow[t]{2}{*}{ III } & A. B. & 0 & 0 & 0,6 & 0,5 & - & 0,6 & 0,2 & 0,3 & 0,3 \\
\hline & H. T. & 0 & 0 & 0,2 & 0,1 & 1,2 & - & 1,0 & 0,1 & 3,4 \\
\hline
\end{tabular}

faßte die Gruppe mit vermehrter Resorption (Gruppe I) Patienten mit Resorptionsmittelwerten über $2 \%$ der applizierten Dosis ${ }^{46} \mathrm{Ca}$ im Liter Serum. Die Gruppe mit normaler Resorption (Gruppe II) zeigte Mittelwerte zwischen $0,7-1,9 \%$ und bei Gruppe III, Patienten mit verminderter Resorption lagen die Werte unter $0,7 \%$ appliziertes ${ }^{46} \mathrm{Ca} / \mathrm{l}$ Serum.

Nach vorheriger Gabe einer Ca-reichen Kost (Tab. 3). sind die Einzel- und Mittelwerte deutlich erhöht. So beträgt der Resorptionsmittelwert für das Gesamtkollek- tiv nach Ca-reicher Kost 2,2\% gegenüber 1,6\% applizierte ${ }^{46} \mathrm{Ca} / \mathrm{l} \mathrm{Serum} \mathrm{im} \mathrm{Versuch} \mathrm{mit} \mathrm{Ca-armer} \mathrm{Diät.} \mathrm{Entspre-}$ chend der insgesamt gesteigerten Resorption wurden nach Ca-reicher Kost für Gruppe I ab 3\%, für Gruppe II zwischen $1-2,9 \%$ und für Gruppe III bis 1,5\% applizierte ${ }^{46} \mathrm{Ca} / \mathrm{l}$ Serum festgelegt.

Die zeitliche Änderung der prozentualen Anteile der applizierten Menge ${ }^{46} \mathrm{Ca} / \mathrm{l}$ Serum ist in Abbildung 1 und 2 für die beiden Versuchsbedingungen anhand der Mittelwerte für die oben gekennzeichneten Gruppen aufge- 


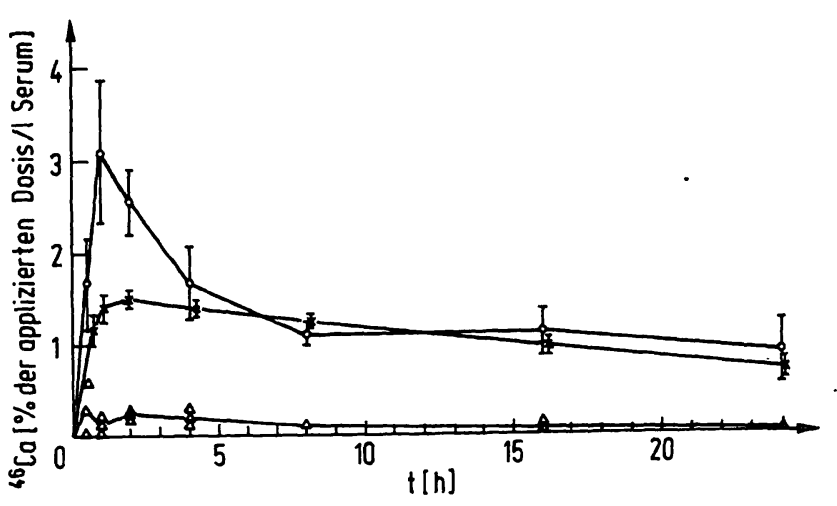

Abb. 1. Zeitliche Änderung der prozentualen Anteile des applizierten ${ }^{46} \mathrm{Ca} / 1$ Serum nach oraler Zufuhr von stabil markiertem $\mathrm{Ca}$ im Anschluß an eine 3tägige Ca-arme Diät. Es sind Patientengruppen mit normaler $(x-x)$, vermehrter $(\bullet-\bullet)$ und verminderter $(\Delta-\Delta)$ Resorption aufgetragen.

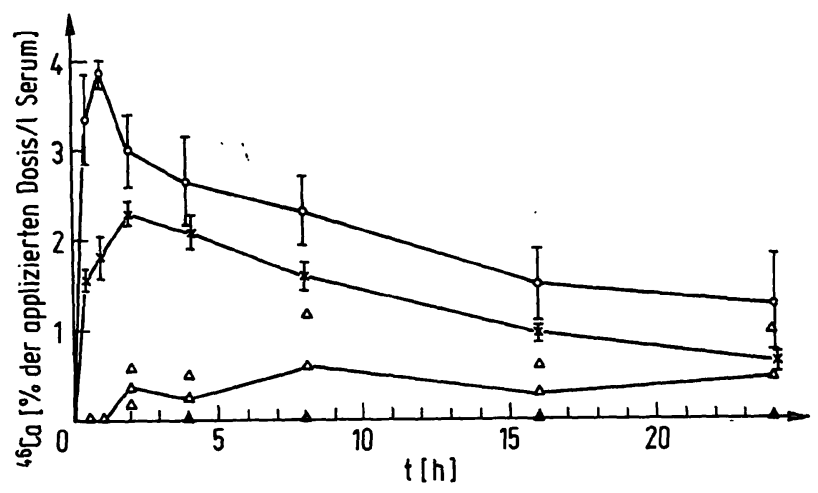

Abb. 2. Zeitliche Änderung der prozentualen Anteile des applizierten ${ }^{46} \mathrm{Ca} / 1$ Serum nach oraler Zufuhr von stabil markiertem $\mathrm{Ca}$ im Anschluß an eine 4tägige Ca-reiche Kost. Es sind Patientengruppen mit normaler $(x-x)$, ver-

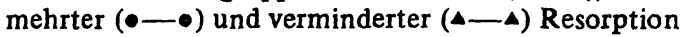
aufgetragen.

tragen. Dabei wird sichtbar, daß sich die mittleren Resorptionswerte zwischen Gruppe I und II nur innerhalb der ersten 2 Stunden deutlich voneinander unterscheiden.

Die Ausscheidung von ${ }^{46} \mathrm{Ca}$ im Urin erfolgte bei den einzelnen Patienten nach unterschiedlichen Zeitintervallen. Die höchsten Anteile des resorbierten ${ }^{46} \mathrm{Ca}$ waren in den Urinportionen zwischen 2 und 12 Stunden nach Einnahme des Isotopengemischs meßbar. Die nach 24 Stunden im Urin ausgeschiedene Menge an ${ }^{46} \mathrm{Ca}$ schwankte zwischen $0,45-5,7 \%$ der applizierten Dosis nach Ca-armer Kost und zwischen 0,24 und 6,3\% nach Ca-reicher Diät. Weder anhand der Gesamtausscheidung über 24 Stunden an ${ }^{46} \mathrm{Ca}$ noch anhand von Einzelportionen ließ sich eine Korrelation zu den oben gekennzeichneten drei Gruppen herstellen.

\section{Diskussion}

Der hier gewählte Weg, das ${ }^{46} \mathrm{Ca}$ nach Aktivierung im Reaktor erst 5 Tage nach Bestrahlungsende über des Gammastrahler ${ }^{47} \mathrm{Sc}$ zu messen, erwies sich als wesentlich einfacher und zudem präziser als die direkte Messung des zunächst entstehenden ${ }^{47} \mathrm{Ca}(8)$, da bei diesem Verfahren die Radioaktivität des ${ }^{24} \mathrm{Na}$ nicht mehr stört. Eine vorherige Abtrennung des $\mathrm{Na}$ würde eine zusätzliche Fehlerquelle darstellen. Die Größe der bei dem von uns gewählten Verfahren noch mit Sicherheit voneinander unterscheidbaren Resorptionsgröße ist neben einer sorgfältigen Probenentnahme und Probenbehandlung abhängig vom Anreicherungsfaktor des ${ }^{46} \mathrm{Ca}$, von der Neutronenflußdichte, von der Bestrahlungs- und Abklingzeit sowie von der Meßdauer und der Meßapparatur. Bei den von uns gewählten Bedingungen, d. h. einer Neutronenflußdichte von circa $5 \times 10^{13} \mathrm{n} /$ $\mathrm{cm}^{2} \cdot \mathrm{s}$, einem handelsüblich erhältlichen Calcium mit einem Anreicherungsgrad von z. B. $28,7 \%$ für ${ }^{46} \mathrm{Ca}$, einer Zufuhr von z. B. 0,36 mg stabil markierten CaIsotopengemischs, einer Bestrahlungszeit von 5 Minuten, einer Abklingzeit von 5 Tagen, einer Meßzeit von 2 Minuten und einer Messung mit einem $\mathrm{Ge}(\mathrm{Li})$-Detektor in Verbindung mit einem Vielkanalimpulshöhenanalysator sind noch $0,1 \%$ der applizierten Dosis unterscheidbar, beziehungsweise reproduzierbar meßbar (13).

Anhand der beschriebenen Methode ist es möglich, $\mathrm{Ca}$ Resorptionsuntersuchungen auch nach Zufuhr eines stabil markierten Ca-Isotops reproduzierbar durchzuführen. Die von uns arbitrarisch vorgenommene Einteilung in Gruppen mit vermehrter, normaler und verminderter Resorption stimmt weitgehend mit dem jeweiligen klinischen Krankheitsbild überein. (siehe Tab. 1). Nur bei dem Patienten H. T. mit der klinischen Diagnose endogene Depression fehlt aus klinischer Sicht eine Ursache für die verminderte Ca-Resorption. Ein Untersuchungsfehler kann ausgeschlossen werden, da in beiden Versuchen gleich niedrige Resorptionswerte gemessen wurden. Der Serum-Ca-Spiegel lag bei diesem Patieriten vor und während des Versuchs mit $2,15 \mathrm{mmol} / \mathrm{lim}$ unteren Normbereich. Möglicherweise lag bei diesem Patienten ein nicht erkannter Hypoparathyreoidismus vor, bei dem Krankheitsbilder mit psychischer Symptomatik vorkommen können. Dabei handelt es sich entweder um Störungen der Affektivität beim sogenannten endokrinen Psychosyndrom (14) oder um Bilder aus dem Formenkreis der organischen Psychosyndrome (15).

Auffällig ist weiterhin, daß die 3 Patienten mit der klinischen Diagnose Tetanie vermehrt Ca resorbierten. Nur einer dieser Patienten lag mit einem Serum-CaWert von 2,00 mmol $/ 1$ unterhalb des Normbereichs. Zur besseren Charakterisierung des Tetanietyps und damit zur gezielten Anwendung einer Therapie ist deshalb auch bei allen Formen einer echten Tetanie die Durchführung eines Ca-Resorptionstestes zu fordern. 
Die gefundenen Resorptionsmaxima liegen bei 1 bis 2 Stunden nach Ca-Einnahme und sinken ab der 4. Stunde deutlich ab. Die vorliegenden Daten zeigen, daß Messungen nach der 4-Stundengrenze eine Unterscheidung von Patienten mit normaler, vermehrter und verminderter Resorption nicht mehr gestatten. Eine Resorptionsbestimmung über 24 Stunden ist somit unnötig und bringt kaum neue Erkenntnisse, wenn man nicht Kompartmentanalysen anstrebt. Ebenso ist anhand der Urinausscheidung an ${ }^{46} \mathrm{Ca}$ eine Einteilung von Patienten in die drei verschiedenen Resorptionsgruppen nicht möglich.

Die Ca-Resorption scheint der oral zugeführten CaMenge proportional zu sein. So nahm der Anteil an Tracer im Serum nach Ca-reicher Kost im Vergleich zur vorherigen Gabe an Ca-armer Diät zu. Im Gegensatz zu den Patienten mit normaler und verminderter Resorption, bei denen die Ca-Anteile nach Erreichen des Höchstwertes nur langsam absank, fielen die Werte in der Gruppe mit vermehrter Resorption so lange schnell ab, bis in etwa das Niveau der Normalgruppe erreicht war. $\mathrm{Ab}$ diesem Zeitpunkt verlaufen beide Kurven annähernd parallel. Nach Zufuhr der Ca-reichen Kost war auch die Regression der Ca-Werte der Patienten mit normaler Resorption beschleunigt. Diese Beobachtung läßt vermuten, daß die Ca-Clearance sowohl bei Gesunden als auch bei Patienten mit Ca-Stoffwechselstörungen von der Resorptionsgröße abhängt. Es ist zu erwarten, daß die $\mathrm{Ca}$-Clearancerate aus dem Serum sofort durch homöostatische Mechanismen verstärkt wird, sobald die aktuelle Ca-Konzentration im Serum eine gewisse Grenze überschreitet. Es ist deshalb für künftige Untersuchungen von Bedeutung, daß vor Testbeginn eine Standardisierung der Ca-Zufuhr eingehalten wird, da nur dann gefundene Meßwerte miteinander verglichen werden können. Die Aussagekraft dieses Testes in bezug auf die Resorption ist nach Einhalten einer Ca-reichen Diät nicht größer als nach Verabreichung einer $\mathrm{Ca}$ armen Kost.

Für künftige klinische Untersuchungen mit angereichertem stabilen ${ }^{46} \mathrm{Ca}$, die vorwiegend Säuglingen, Kindern und Schwangeren vorbehalten bleiben sollen, wird anhand dieser Ergebnisse folgendes vereinfachtes Vorgehen empfohlen: nach 3-tägiger standardisierter Diät wird das stabil markierte ${ }^{46} \mathrm{Ca}$ oral verabreicht und innerhalb der ersten 2 Stunden 2-bis 3-mal venöses Blut entnommen. Aus den gemessenen prozentualen Anteilen des applizierten ${ }^{46} \mathrm{Ca} / 1$ Serum wird ein Mittelwert gebildet. Auf eine Sammlung des Urins wird verzichtet. Anhand der ermittelten Werte für das stabil markierte $\mathrm{Ca}$, gemessen in Prozent der applizierten Dosis pro 1 Serum, wird eine Zuordnung zu den 3 verschiedenen Resorptionsgruppen durchgeführt. Einer Untersuchung von Ca-Stoffwechselstörungen bei den oben genannten Patientengruppen soll te somit nichts mehr im Wege stehen.

\section{Literatur}

1. Szymendera, J., Heaney, R. P. \& Saville, P. D. (1972), J. Lab. Clin. Med. 79, 570-578.

2. Birge, S. J., Peck, W. A., Berman, M. \& Whedon, G. D. (1969), J. Clin. Invest. 48, 1705-1713.

3. Shimmins, J., Smith, D. A., Aitken, M., Linslay, G. S., Orr, J. S. \& Gillespie, F. C. (1971), Calc. Tiss. Res. 6, $301-315$.

4. Hesch, R. D., Gerlach, W., Henning, H. V., Emrich, D., Scheler, F. \& Kattermann, R. (1972), Deut. Med. Wochenschr. 97, 1735-1742.

5. Avioli, L. V., McDonald, J. E., Singer, R. A. \& Henneman, P. H. (1965), J. Clin. Invest. 44, 128-139.

6. McPherson, D. (1965), Acta Ortop. Scand. Suppl. 78, 34-61.

7. Kasperek, K. \& Ritzl, F. (1966), Interner Bericht KFA Jülich Me-4.

8. Bethard, W. F., Schmitt, R. A., Olehy, D. A. Kaplan, S. A., Ling, S. M., Smith, R. H. \& Dalle Molle, E. (1967), IAEA-Symposium Amsterdam, Paper SM-91/65.

9. Kasperek, K., Ritzl, F. \& Stockhausen, W. (1968), Strahlentherapie 136, 71-75.

10. Kahn, B., Straub, C. P., Robbins, P. J., Wellman, H. N. Seltzer, R. A. \& Telles, N. C. (1969), Pediatrics 43, 651-667.

11. Barltrop, D. \& Sutton, A. (1972), IAEA Symposium Bled, Paper SM-91/65.

12. Hallmann, L. (1966), Klinische Chemie und Mikroskopie, 10. Aufl. Verlag Thieme, Stuttgart.

13. Ritzl, F. \& Kasperek, K. (1967), Elektromedizin 12, 236239.

14. Bleuler, M. (1954), Endokrinologische Psychiatrie, Verlag Thieme, Stuttgart.

15. Denko, J. \& Kaebling, R. (1962), Acta Psychiat. Scand. 38, Suppl 164.
Anschrift der Verfasser:

Dr. M. Persigehl

Abt. Radiologie

der RWTH Aachen

51 Aachen

Goethestraße 27/29

Für die übrigen Verfasser:

Prof. Dr. L. E. Feinendegen Institut für Medizin der

KFA Jülich

517 Jülich

Postfach 365 
